\title{
Kinematic strangeness production in cluster hadronization
}

\author{
Cody B. Duncan ${ }^{1,2, a}$, Patrick Kirchgaeßer ${ }^{2}$ \\ ${ }^{1}$ School of Physics and Astronomy, Monash University, Clayton, VIC 3800, Australia \\ ${ }^{2}$ Institute for Theoretical Physics, Karlsruhe Institute of Technology, 76128 Karlsruhe, Germany
}

Received: 30 November 2018 / Accepted: 20 December 2018 / Published online: 28 January 2019

(C) The Author(s) 2019

\begin{abstract}
We present a modification to the non-perturbative strangeness production mechanisms in the Monte-Carlo event generator Herwig in order to make the processes more dynamic and collective. We compare the model to a series of observables for soft physics at both LEP and LHC.
\end{abstract}

\section{Introduction}

The non-perturbative elements of simulating LHC events remain an active area of research in light of recent ALICE and CMS data [1,2]. Signs of strangeness enhancement and collective effects in high multiplicity events respectively have inspired several phenomenological models, ranging from interacting strings [3,4], to relativistic hydrodynamics [5], to tweaks to the existing multiple parton interaction mechanisms [6] and colour reconnection [7,8] models. Monte Carlo event generators [5,9-11] provide a useful testing ground for these models.

Arguably the most successful models of hadronization which try to reproduce strangeness enhancement in highmultiplicity events are rooted in the physics of collectivity, where the dense environment of high multiplicity events leads to more complicated systems which interact with one another. Heavy ion event generators typically prefer a hydrodynamic viewpoint, where the quark-gluon plasma acts as a perfect fluid, changing the dynamics of hadronization. High-energy $p p$ event generators tend to use sophisticated iterations of the more conventional proton collision techniques, such as the DIPSY rope model where several overlapping Lund strings [12] combine into a higher-representation colour field, which then may enhance strangeness production and may also shove each other transversely outwards, mimicking the fluid behaviour of quark-gluon plasma. Another model [13] has attempted to use a thermodynamics inspired

a e-mail: cody.duncan@monash.edu route to string fragmentation and was able to explain a harder transverse momentum spectrum for heavier particles.

Herwig [9] has recently developed a new model for colour reconnection, where baryonic clusters were allowed to be produced in a geometric fashion [8], in an attempt to explain the results of [1]. The model was able to create heavier hadrons, and in particular more baryons, but in order to better describe the data, the non-perturbative gluon splitting mechanism was allowed to produce $s \bar{s}$ pairs as well as the default lighter species. However, the production weight was simply set to a flat number, tuned to Minimum Bias events at the LHC. In this paper, we will mainly focus on the fundamental mechanisms of strangeness production in cluster hadronization, namely the production rate of $s \bar{s}$ pairs during non-perturbative gluon splitting, cluster fission, and cluster decay. In doing so, we are taking the first steps to a rework of strangeness production in the Herwig hadronization phase. A full model would also need to consider colour reconnection, since this rearranges the colour topology and thus the mass distribution inside an event, affecting the scaling that we are interested in studying.

In this study, we aim to introduce a simple dynamic model of strangeness production in Herwig, in which each nonperturbative production stage uses the kinematic information of the relevant surrounding colour-singlet system. After reviewing the current mechanisms of hadronization in Sect. 2, we perform two separate tunes to a number of light strange meson observables for LEP and LHC Minimum Bias events in Sect.3. We show that the tuned current strangeness production parameters are drastically different between the two collider types, and propose a mass-based scaling for the relevant production weights in Sect. 4, comparing two different mass-like measures to scale the probability. In Sect. 5, we tune our new model and compare the results with the old model in Herwig, as well as perform a comparison to the default Lund string model in Pythia [10] with the Monash tune [14]. We briefly summarize the work and possible future avenues for research in Sect. 6. 


\section{The Herwig Hadronization Model}

To accurately describe a full QCD event, one must be able to model the non-perturbative physics contributions, e.g. hadronization of individual quarks \& gluons from the parton shower and the multiple parton interactions to form coloursinglet hadrons.

Figure 1 sketches a schematic event, focusing on the final state. After generating a hard matrix element for the event, Herwig performs a parton shower, producing a number of soft and collinear partons. After the parton shower reaches $\mathcal{O}(1) \mathrm{GeV}$, the hadronization phase of simulation occurs. In Herwig, the hadronization model is the cluster model [15], based on the colour preconfinement [16] property from the angular-ordered parton shower. A cluster can be considered to be a highly primordial, excited colour-singlet $q \bar{q}$ pair.

There are several parts to the hadronization model in Herwig, in the following algorithmic order:

- Non-perturbative gluon splitting,

- Colour reconnection,

- Cluster fission,

- Cluster decay to hadron pairs,

- Unstable hadron decays.

In Fig. 1, we have omitted colour reconnection since this step simply changes the colour topology of the event, not the content of the clusters. While modifying the colour reconnection algorithm would have a non-trivial impact on the later stages of hadronization, namely cluster fission and decay, it is outside the scope of this paper, but these correlations will be studied and addressed in future work. Since the scope of this

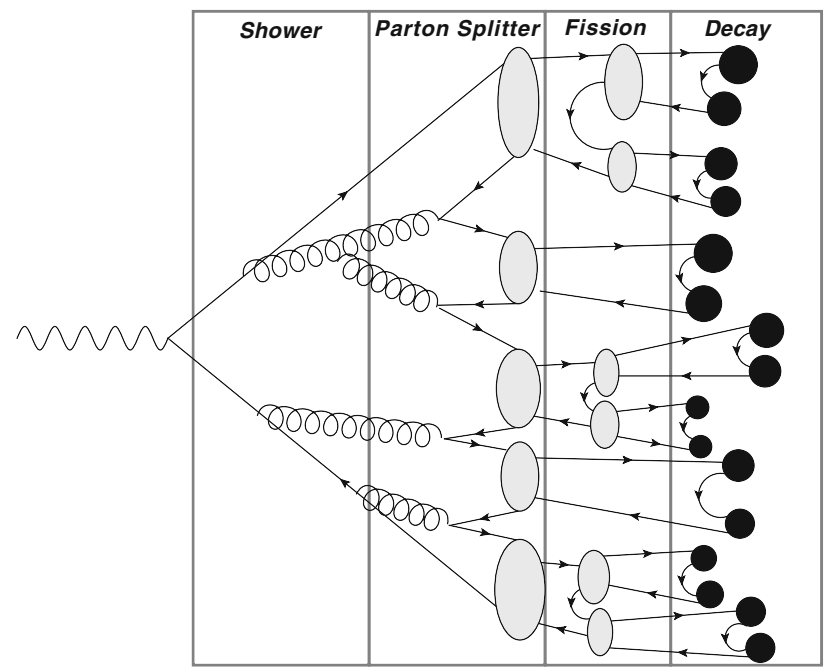

Fig. 1 Figure of a simplified event where we show the major stages of hadronization after the parton shower that can contribute to nonperturbative strangeness production. Grey ellipses are clusters, while black are hadrons project is mainly focused on light strange hadron production, we tune predominately to pion and kaon observables. We will also ignore unstable hadron decays for the purposes of this paper.

The three other listed stages in hadronization are each allowed to contribute to the overall strangeness in the event, since they each produce new $q \bar{q}$ pairs. We briefly recall the details of each step as presented in depth in [9].

\subsection{Non-perturbative gluon splitting}

Once the parton shower ends, all gluons undergo a nonperturbative splitting into $q \bar{q}$ pairs. The species of the pair is determined by a given weight, e.g. in the tune from [8] the weights of up, down, and strange are $2: 2: 1$. The default version of Herwig does not allow for strangeness production at this step, only $u \bar{u}$ and $d \bar{d}$ pairs. The only constraint on the gluon splitting is that the gluon mass is at least twice the constituent mass of the species in question, and the gluons are split isotropically.

After all the gluons in an event have been split, nearest neighbours in momentum space are most likely to be nearest neighbours in colour space [16], and clusters are formed from the momentum-space neighbouring $q \bar{q}$ pairs, with a mass distribution decoupled from the hard scattering process that created them.

\subsection{Cluster fission}

Exceptionally heavy clusters are allowed to fission into two lighter, less excited clusters if the mass $M$ of the original cluster satisfies the condition:

$M^{p} \geq q^{p}+\left(m_{1}+m_{2}\right)^{p}$,

where $p$ and $q$ are parameters that control the fissioning rate criteria, and $m_{1,2}$ are the parton masses of the heavy cluster. In Herwig, $p$ is given separate values for light quarks $(u, d, s)$, charm, and bottom. The light quark weights are further subdivided, and strangeness is suppressed by a flat weight. $q$ has a similar divide between the quark species.

After selecting clusters to fission, the cluster fissioner produces a $q \bar{q}$ pair from the light quarks with a fixed weight, distinct values for each flavour of quark (bar top), and diquarks. Each parton from the pair go into a separate cluster, giving the new pair of clusters a mass distribution of:

$M_{i}=m_{i}+\left(M-m_{i}-m_{q}\right) R_{i}^{1 / w}$,

where $w$ is the splitting parameter that controls the rate of splitting for clusters containing different species of quarks. 


\subsection{Cluster decay}

The last stage of cluster-based physics is at the cluster decay level, in which clusters decay into excited hadrons. Given a cluster with constituents $q_{1}, \bar{q}_{2}$, the weight for producing hadrons $h_{a}=q_{1} \bar{q}, h_{b}=q \bar{q}_{2}$, where $q$ denotes a quark or diquark species, is given by:

$\mathcal{W}\left(h_{a}, h_{b}\right)=P_{q} w_{a} s_{a} w_{b} s_{b} p_{a, b}^{*}$,

where $P_{q}$ is the production weight for the given quark or diquark species, $w_{i}$ are the weights for the relevant hadron production, and $s_{i}$ are the suppression factors for the corresponding hadrons. The final factor in the weight is the twobody phase space factor that controls how readily the cluster can decay into the two chosen hadrons.

\subsection{Herwig strangeness parameters}

The Herwig parameters that control non-perturbative strangeness production are the gluon splitting weight SplitPwtSquark, and the cluster fission \& decay weight - PwtSquark. In the original model, cluster fissioning and cluster decaying are controlled by the same parameter. The first step in our understanding of the different contributions is to disentangle cluster fission from cluster decay and introduce one additional parameter which controls the production of a $s \bar{s}$ pair during cluster fission - FissionPwtSquark. The decay parameter remains the same.

\section{Tuning of the existing model}

In this section we tune the parameters for strangeness production of the existing model first to LEP and then to LHC data. Hadronization models are typically tuned to LEP data if they do not rely on $p p$-specific event topology, e.g. multiple parton interactions and their effects on colour reconnection, since LEP provides a clean QCD final state environment which imposes relatively strict constraints on what one's hadronization model is allowed to do.

The tuning is achieved by using the Rivet and Professor frameworks for Monte Carlo event generators [17, 18]. In order to understand the overall effects of strangeness production on different stages of the event generation, we keep all other hadronization parameters that were previously tuned to LEP data at their default values $[9,19]$. In the first tune (TUNE1), we only consider the effects of the parameters that are directly responsible for strangeness production as explained in Sect. 2.
In a second tuning attempt (TUNE2), we introduce the new parameter for the cluster fission stage. Tuning these 3 different parameters will allow us to study the phases of strangeness production during event generation and will shed light on the differences between LEP and LHC.

We note that this section is an extended part of the introduction to visualize and highlight the effects of the aforementioned different parameters and to see at which stage non-perturbative strangeness production is preferred.

\subsection{LEP tuning}

For the tuning to LEP data, the following observables from ALEPH [20,21], DELPHI [22], SLD [23] and PDG hadron multiplicities [24], which represent a good description of event shapes and $\pi, K$ multiplicities, were used with equal weights:

- Mean charged multiplicities for rapidities $|y|<1.0$, $|y|<1.5$ and $|y|<2.0$

- $K^{0}$ spectrum

- Mean $\pi^{0}$ multiplicty

- Mean $K_{S}+K_{L}$ multiplicity

- Mean $K^{0}$ multiplicity

- Mean $\pi^{+} / \pi^{-}$multiplicty

- Mean $K^{+} K^{-}$multiplicity

- Ratio (w.r.t $\pi^{ \pm}$) of mean $K^{ \pm}$multiplicity

- Ratio (w.r.t $\pi^{ \pm}$) of mean $K^{0}$ multiplicity

- $K^{ \pm}$scaled momentum

The resulting parameter values for the two different tunes are listed in Table 1.

While being able to describe all the considered LEP data on equally good footing, we improve the simulation of the observables which were considered in the tuning procedure. TUNE2 gives better agreement to the data, at least with respect to the $K^{ \pm}$multiplicity, highlighting the necessity to disentangle the cluster fission and cluster decay parameters. The corresponding plots are shown in Fig. 2, where we compare the default version with our two new tunes.

Table 1 Results of the parameter values for strangeness production at the different stages of the event generation (LEP). In both default Herwig and TUNE1, cluster fission and decay have the same parameter. In TUNE2, they are allowed to be different, but the tuning procedure returned equal values. In default Herwig, there is no $g \rightarrow s \bar{s}$ option

\begin{tabular}{lccc}
\hline LEP & Default & TUNE1 & TUNE2 \\
\hline Gluon Splitting & - & 0.24 & 0.19 \\
Cluster Fission & 0.66 & 0.53 & 0.69 \\
Cluster Decay & 0.66 & 0.53 & 0.69 \\
\cline { 2 - 3 } & \multicolumn{2}{c}{0.} \\
\hline
\end{tabular}



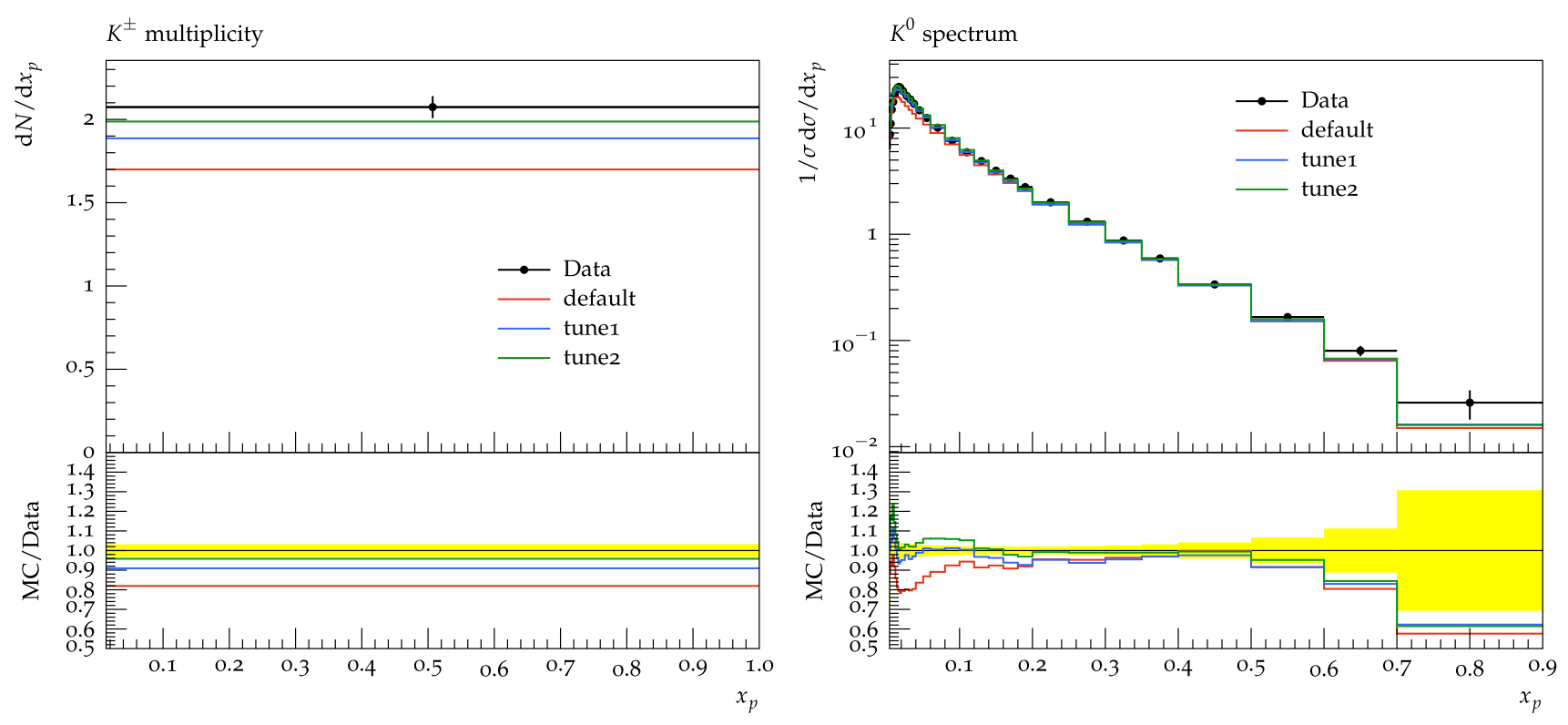

Fig. 2 Measurement of $K^{ \pm}$multiplicities at SLD [23] and $K^{0}$ spectrum as measured at ALEPH [20] for $\sqrt{s}=91.2 \mathrm{GeV}$. We show a comparison between the default Herwig model and our two different tunes

\subsection{LHC tuning}

For the tuning to LHC data, we solely focus on identified particle distributions which were measured at ALICE [25] and CMS [2]. We limit the tuning to a center of mass energy of $\sqrt{s}=7 \mathrm{TeV}$ due to the lack of suitable available Rivet analyses at higher energies. The following observables were considered in the tuning procedure with equal weights:

- $K^{+}+K^{-}$yield in INEL pp collisions at $\sqrt{s}=7 \mathrm{TeV}$ in $|y|<0.5$

- $K / \pi$ in INEL pp collisions at $\sqrt{s}=7 \mathrm{TeV}$ in $|y|<0.5$

- $K_{S}^{0}$ rapidity distribution at $\sqrt{s}=7 \mathrm{TeV}$

- $K_{S}^{0}$ transverse momentum distribution at $\sqrt{s}=7 \mathrm{TeV}$

The resulting parameter values are shown in Table 2.

The outcome of the tuning procedure is shown for the $p_{T}$ distribution of $K^{+}+K^{-}$yields and the $K / \pi$ ratio in Fig. 3 .

Again the retuning of the default model with the incorporation of an additional independent parameter at the cluster fission stage improves the description of the considered observables significantly.

\subsection{Summary}

The general approach in tuning a hadronization model is to tune the parameters to LEP data and then assume it is able to describe LHC observables as well since hadronization is assumed to factorize and should not depend on the process involved.
Table 2 Results of the parameter values for strangeness production at the different stages of the event generation (LHC). In both default Herwig and TUNE1, cluster fission and decay have the same parameter, while in TUNE2 they are allowed to be different. In default Herwig, there is no $g \rightarrow s \bar{s}$ option

\begin{tabular}{lccc}
\hline LHC & Default & TUNE1 & TUNE2 \\
\hline Gluon Splitting & - & 0.95 & 0.95 \\
Cluster Fission & 0.66 & 0.05 & 0.02 \\
Cluster Decay & 0.66 & 0.05 & 0.25 \\
\cline { 2 - 3 } & \multicolumn{3}{c}{} \\
\hline
\end{tabular}

The main difference between LEP and LHC is the denser hadronic environment one encounters due to multiple parton interactions and therefore also the enhanced effect of colour reconnections on the distribution of final state particles. Be that as it may, we believe that the probability to produce strangeness e.g at the stage of non-perturbative gluon splitting should be a universal parameter and be independent of the process in question.

Since the data shows that clearly different parameter values are preferred at LHC and LEP the approach to have a single valued probability is not suited for the description of both LHC and LEP observables. It may capture the average effect but it does not allow for fluctuations on an event-byevent basis. We tackle this problem by assuming that the rate at which strangeness is produced depends on the hadronic density of the immediate environment, which will be discussed in the next section. 

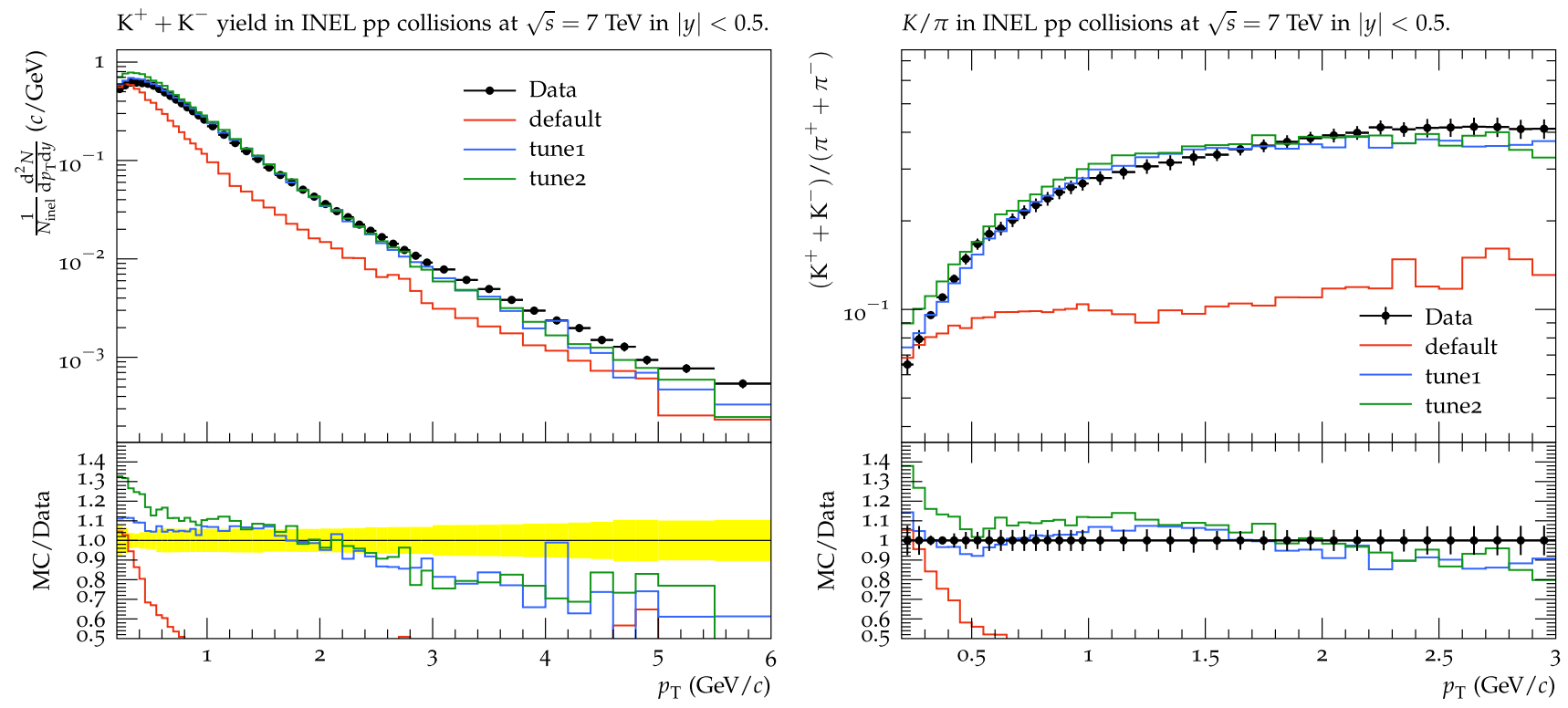

Fig. 3 Transverse momenta spectra for $K^{+}+K^{-}$and $K / \pi$ ratio as measured by ALICE[25] at $\sqrt{s}=7 \mathrm{TeV}$ in the central rapidity region. We show a comparison between the default Herwig model and our different tunes.

\section{Kinematic strangeness production}

As mentioned above, the various splitting probabilities and weights are flat numbers tuned to data, without any considerations for the topology of a given event. In order to have a more dynamic picture, where the splitting probabilities depend on the environment, we choose to scale the weights with respect to colour-singlet masses. The mass of a colour-singlet system at a given phase of hadronization scales the probability for strangeness production up or down, depending on a characteristic mass scale for each step.

As a simple starting point for mass-based power scaling, we replace the flat weights in each of the steps mentioned in Sect. 2 with the following functional form:

$w_{s}(m)^{2}=\exp \left(\frac{-m_{0}^{2}}{m^{2}}\right)$

where $m_{0}^{2}$ is the characteristic mass scale for each phase, and $\mathrm{m}^{2}$ is the total invariant mass of the relevant colour-singlet system. In this work, we will introduce another mass-based measure which replaces $m^{2}$ in the denominator of Eq. 4: the threshold production measure, $\lambda$. We discuss the difference in the two approaches in Sect. 4.3. For now, we will continue to use the total invariant mass as an example in the following sections.

The weights in Eq. 4 are only for strangeness production, and they are relative to the production weights of up and down quarks. In the limit of a very heavy colour-singlet, the rate of producing strangeness will be the same as that of the lighter quarks, while in the low-mass limit, only the lighter quarks will be allowed to be produced. The appeal of an exponential scaling is that this model only introduces one extra parameter to the default model of hadronization in Herwig, and indeed, it does not introduce any extra parameters if one splits the fission and decay parameters. Thus we avoid a proliferation of parameters in our model, and we still have a natural mechanism to allow for event-by-event fluctuations in strangeness production.

The scaling of the production rate in Eq. 4 only applies to $s \bar{s}$ pairs, and not to any diquarks containing strange quarks. Default Herwig does not allow gluons to non-perturbatively split into diquark-diantiquark pairs, nor does it allow these pairs to be produced during cluster fissioning and decay. Diquarks may only be produced as remnants of the incoming baryons, or from baryon-number violating processes [9]. Since diquark species would fundamentally affect the baryon yields, which we are not studying in this work, we leave diquark production considerations to a future rework of baryon production in Herwig.

\subsection{Non-perturbative gluon splitting}

At the end of the shower, instead of immediately splitting the gluons into $q \bar{q}$ pairs with the species determined by their given weights, we instead collect the various colour-singlet systems in the event, what we call pre-clusters. While colour preconfinement dictates that the mass distribution of clusters is independent of the hard energy scale, there are no such constraints on the masses of the colour-singlet pre-clusters. As shown schematically in Fig. 6, a parton shower can produce gluons and quark-antiquark pairs at a perturbative level, 

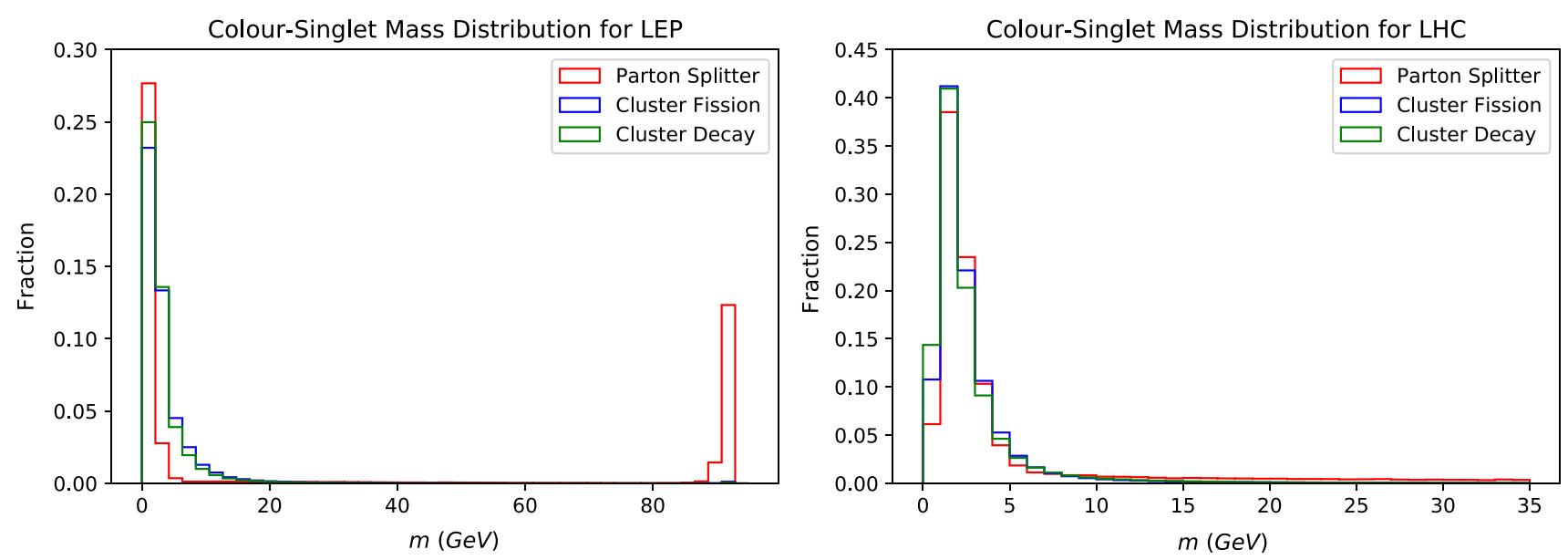

Fig. 4 Mass distributions for colour-singlet systems immediately before the Parton Splitter, Cluster Fissioner, and Cluster Decayer steps in LEP and LHC Minimum Bias events. Note the different mass axis scales
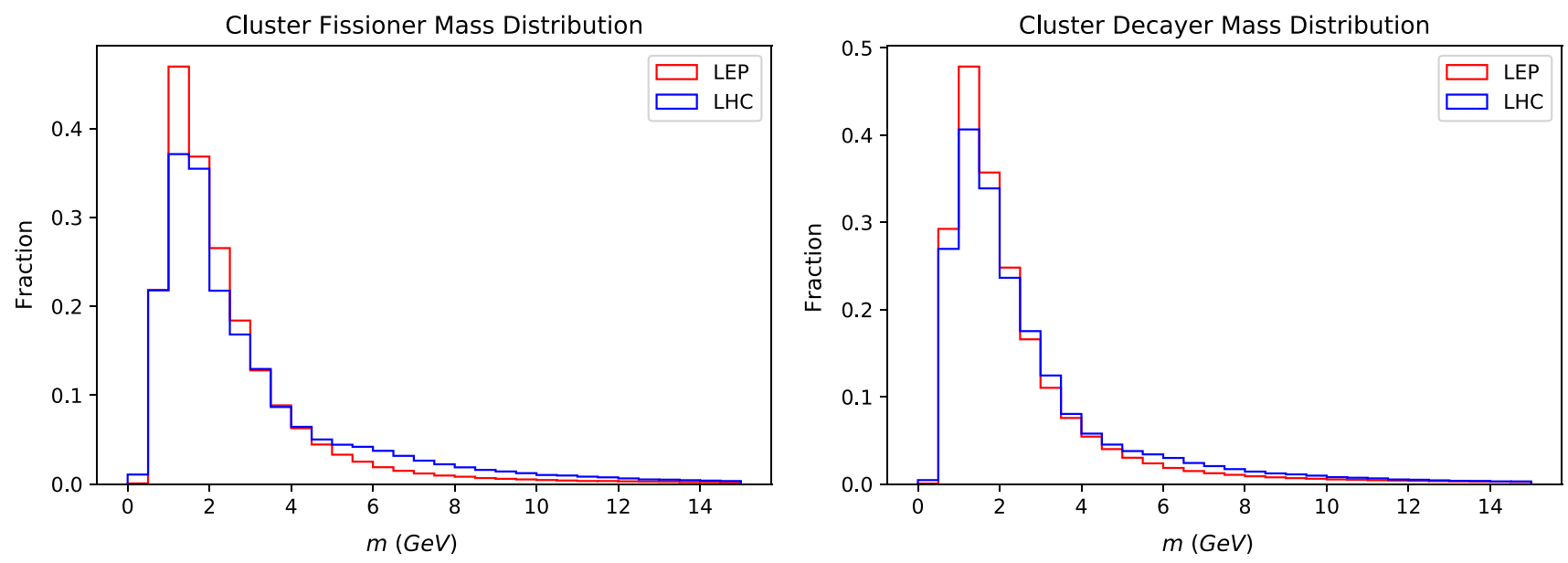

Fig. 5 Comparison of LEP and LHC Minimum Bias mass spectra of clusters immediately before cluster fission and cluster decay

separating the event into a number of different pre-clusters with a variety of masses.

Every gluon in the same pre-cluster will get the same weight, since they belong to the same colour-singlet system, and thus have the same mass measure for strangeness production, but since the species is picked probabilistically, this does not mean that all the gluons will produce strange quark-antiquark pairs. The constraint from default Herwig still applies, namely that even in situations where there is a very heavy pre-cluster, if a gluon cannot access the phase space necessary to split into a $s \bar{s}$ pair, then it will undergo the usual splitting to up or down quarks.

The characteristic mass scale for pre-clusters will unfortunately depend on the type of collider one uses. As shown in Fig. 4, there is a very broad tail for the proton colliders due to the number of pre-clusters that one can produce. This is a by-product of the type of dense and complicated final state environment of high energy hadron colliders. At LEP, there are two peaks for the pre-cluster mass distribution, one at close to $91.2 \mathrm{GeV}$, corresponding to events where there are only gluon emissions from the outgoing $q \bar{q}$ legs from the hard scattering process, and very few colour-singlets fall between the two peaks, due to the simple fact that perturbative gluon splitting is suppressed compared to perturbative gluon emission.

\subsection{Cluster fission and decay}

At the cluster fission and cluster decay level, the coloursinglet is the cluster itself. We allow the characteristic mass scale and characteristic production probability to be different for the two phases. As shown in Fig. 5, the typical cluster masses at the cluster fission and cluster decay stages are roughly similar for both LEP and LHC, which we hope to reflect in the characteristic mass scales for the two tunes. We note that Figs. 4 and 5 are plotted without turning on the exponential scaling, which would change the mass 


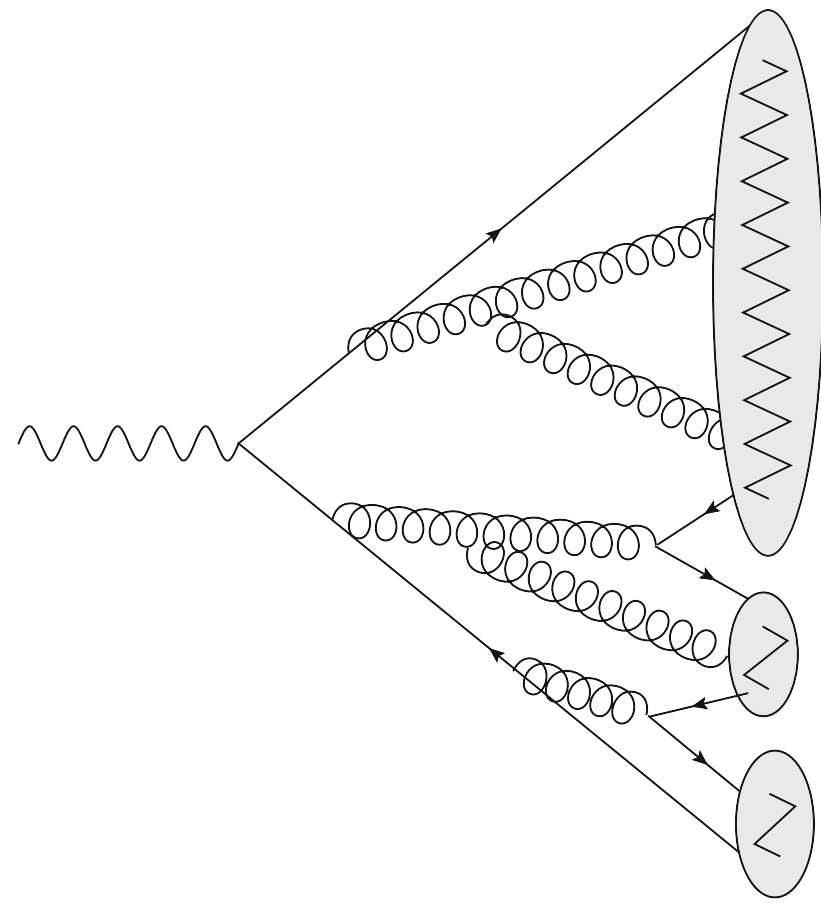

Fig. 6 Schematic topology of colour-singlets that can occur from perturbative gluon and quark shower splitting, before the gluons undergo non-perturbative splitting

distribution slightly, but the figures are benchmarks of the typical colour-singlet total invariant masses.

\subsection{Colour-singlet masses}

In the previous sections we have used the total invariant mass of the colour-singlet systems as the mass measure in Eq. 4, but there are issues with this approach. In using the total invariant mass of a given colour-singlet to scale the strangeness weight,
Table 3 Results for the tuned characteristic mass scales $m_{0}$, in units of $\mathrm{GeV}$, of our new model using the total invariant mass of a colour-singlet object for LEP and LHC tunes respectively

\begin{tabular}{lrr}
\hline Invariant mass & LEP & LHC \\
\hline Gluon Splitting & 97 & 48 \\
Cluster Fission & 3 & 22 \\
Cluster Decay & 23 & 4 \\
\hline
\end{tabular}

we have neglected to take into account the massive nature of the partons in the pre-clusters and clusters. We argue that given two colour-singlets of the same total invariant mass, if one cluster has much heavier endpoints or constituents that the other, then the one with lighter endpoints or constituents should more readily produce $s \bar{s}$ pairs from the vacuum (Fig. 6).

To remove the biasing effects of massive constituents, we have implemented another mass measure:

$\lambda=m_{c s}^{2}-\left(\sum_{i} m_{i}\right)^{2}$,

where $m_{c s}^{2}$ is the total invariant mass of the colour-singlet system, and $m_{i}$ are the invariant masses of the endpoints for pre-clusters or the constituent partons in a cluster.

Gluons are massive in Herwig, but because their masses are used to produce the $s \bar{s}$ pair, we do not include them in the subtraction term. The $\lambda$ measure would replace the massbased denominator in Eq. 4. We have presented the distributions of the $\lambda$ measure for each of the stages in Fig. 7, and a comparison between the distributions of the two mass measures in Figs. 9 and 8 . The $\lambda$ measure has the appealing feature that if one produced a $s \bar{s}$ pair at the gluon splitting level, this extra mass wouldn't propagate extra strangeness enhancement further into the hadronization process.
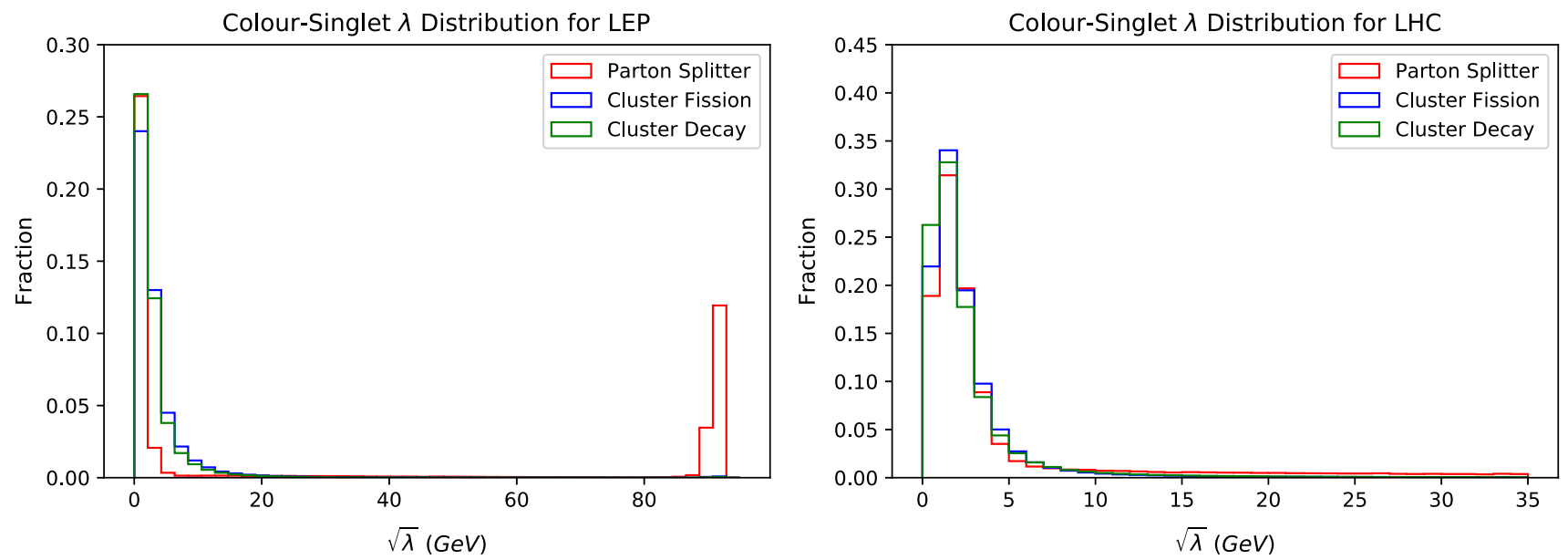

Fig. 7 Threshold mass, $\lambda$, distributions for colour-singlet systems immediately before the Parton Splitter, Cluster Fissioner, and Cluster Decayer steps at LEP events at $91.2 \mathrm{GeV}$ and LHC Minimum Bias events at $7 \mathrm{TeV}$ 

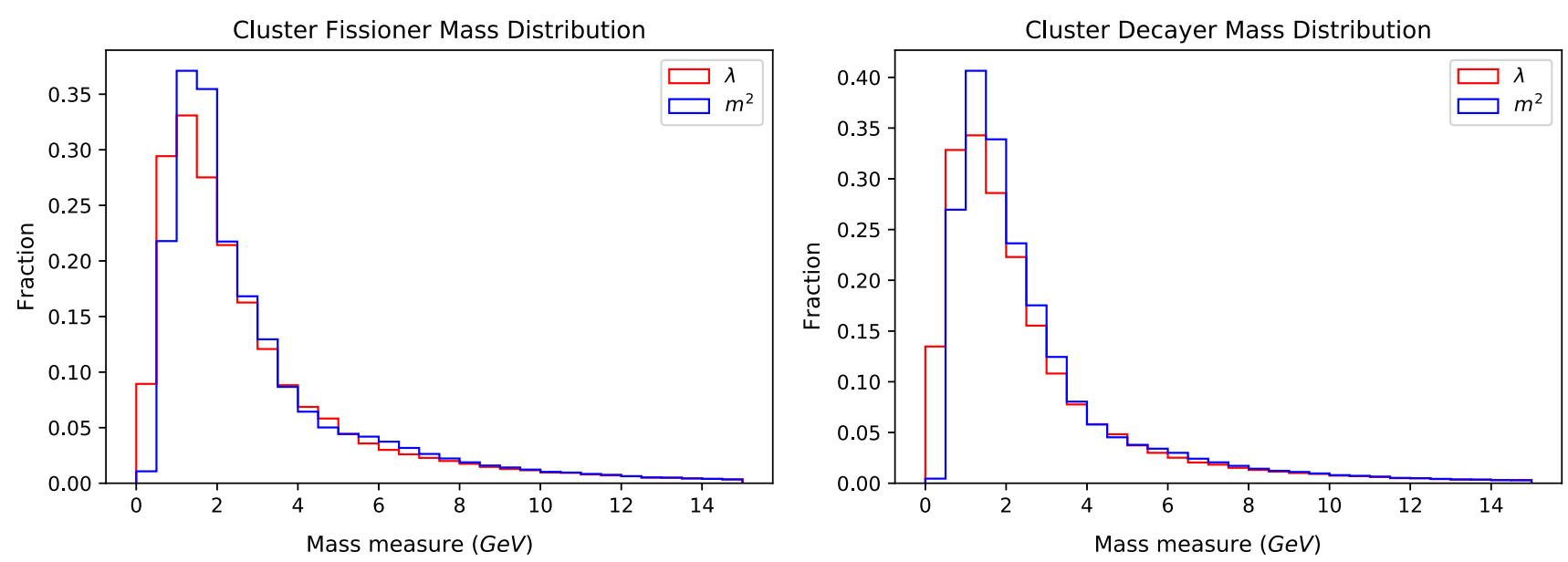

Fig. 8 Comparison of the two different mass measures for the cluster fission and cluster decayer stages respectively for LEP events at $91.2 \mathrm{GeV}$
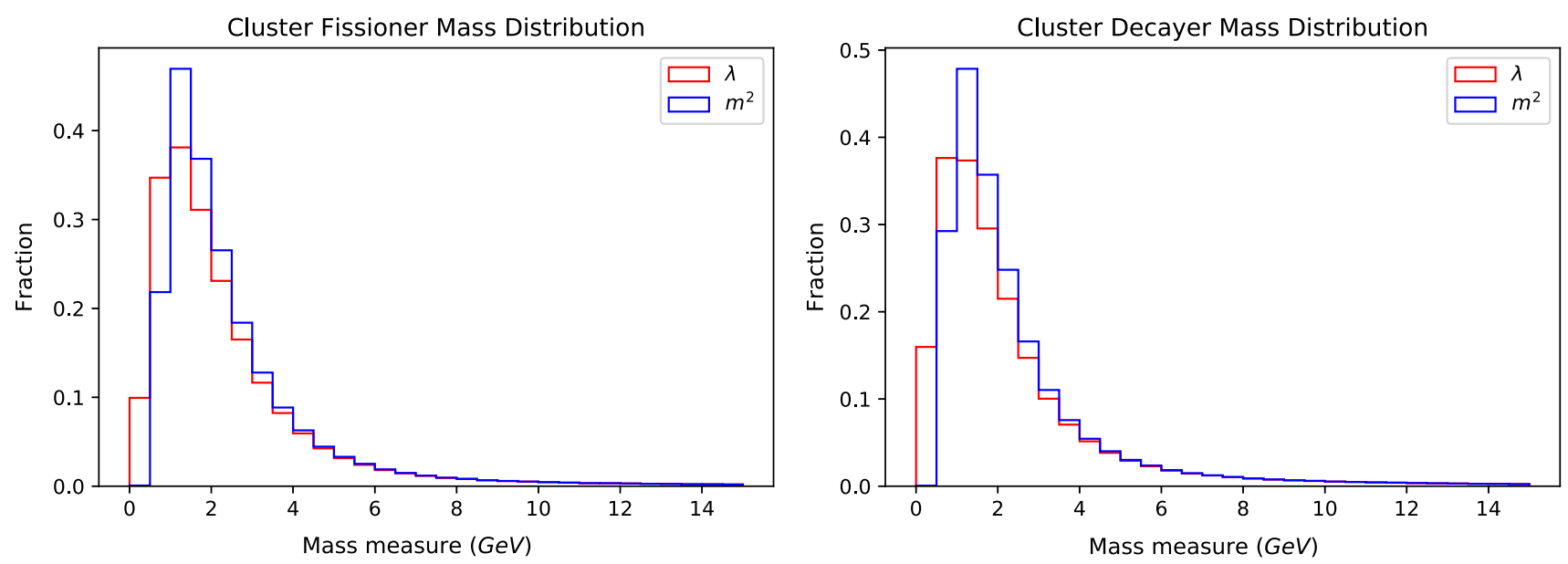

Fig. 9 Comparison of the two different mass measures for the cluster fission and cluster decayer stages respectively for LHC Minimum Bias events at $7 \mathrm{TeV}$

\section{Analysis}

We first tune the 3 parameters of our mass-based scaling model to the same identified strange particle yields at LEP and LHC as in Sect. 3. The new tunable parameters are MassScale (for gluon splitting), FissionMassScale, and DecayMassScale, which are defined by Eq.4. The outcome of the tuning procedure for the relevant parameter values is shown in Tables 3 and 4 for LEP and LHC Minimum Bias, for both the total invariant mass measure and the $\lambda$ measure.

With the three new characteristic mass scales, we are able to improve the description of all observables considered in the tuning especially for LHC observables as shown in Fig. 10, where we compare the two different mass measures after tuning, as well as the Monash tune [14] for Pythia.

Although the simple tuning recommends different values for the usage at LHC and LEP it is also feasible to use the set of parameters obtained from the tuning to LHC data and
Table 4 Results for the tuned characteristic mass scales $m_{0}$, in units of $\mathrm{GeV}$, of our new model using our $\lambda$ measure (defined in Eq. 5) of a colour-singlet object for LEP and LHC tunes respectively

\begin{tabular}{lrr}
\hline$\lambda$ measure & LEP & LHC \\
\hline Gluon Splitting & 72 & 37 \\
Cluster Fission & 4 & 20 \\
Cluster Decay & 16 & 10 \\
\hline
\end{tabular}

still get improved results for LEP observables which was not possible by having a simple flat number as the probability to produce strange quarks as is shown in Fig. 11.

\subsection{Discussion}

The default version of Herwig did not allow for strange production during the gluon splitting stage. By allowing this process, improvements can be seen in all the considered observables. With our new model, there is a more physically motivated dynamic strangeness production mechanism at all stages of the hadronization. 


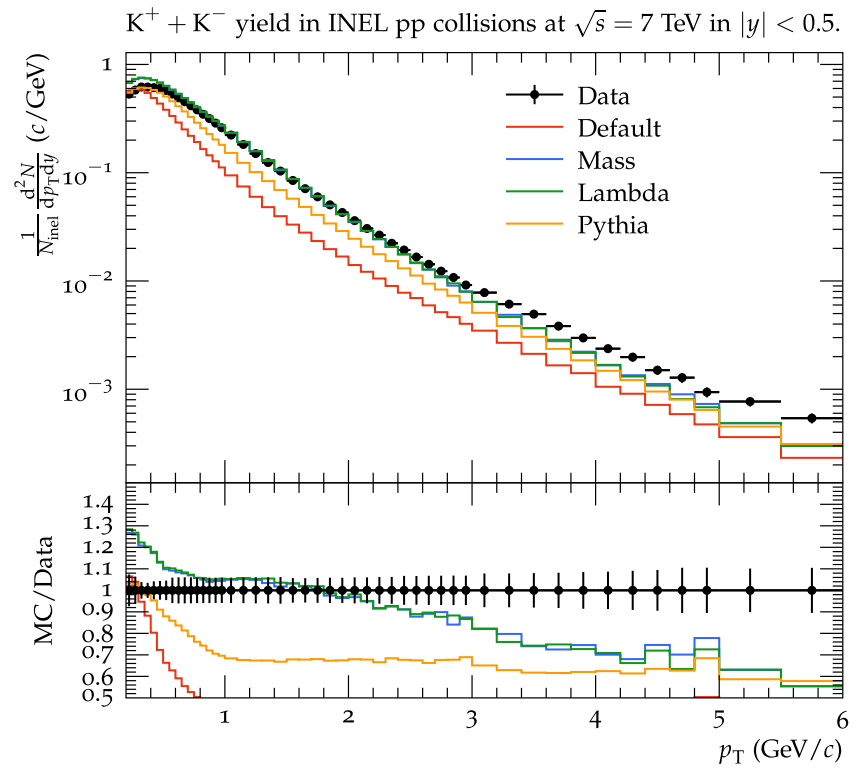

Fig. $10 K^{+}+K^{-}$yield and $K / \pi$ ratio as measured by ALICE [25] at $7 \mathrm{TeV}$. Shown is a comparison between the default version of Herwig (without baryonic reconnection), i.e. static production of strangeness,

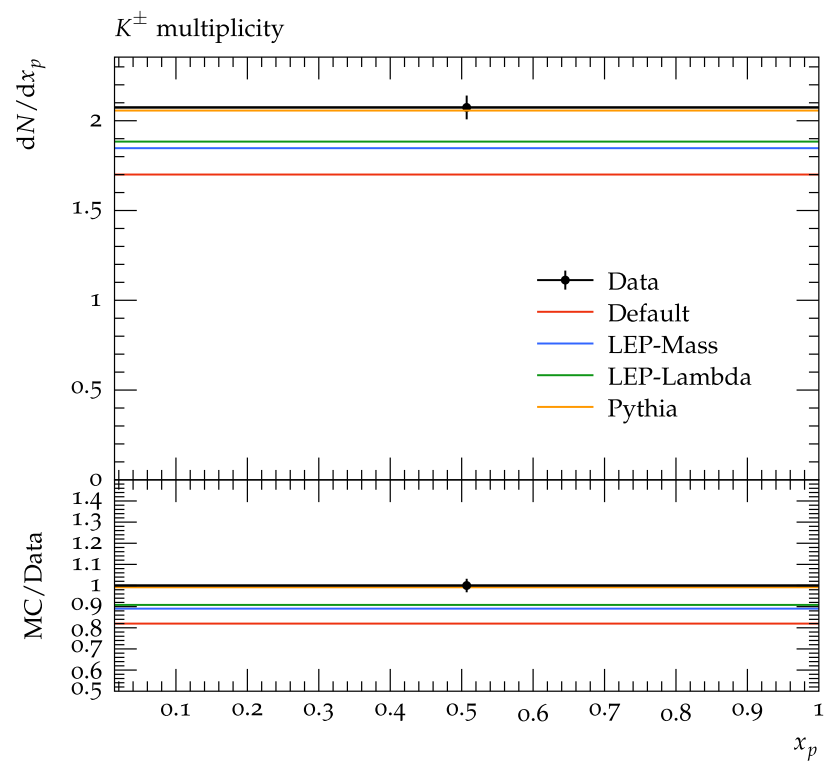

Fig. 11 Measurement of $K^{ \pm}$multiplicities at SLD [23] $\sqrt{s}=$ $91.2 \mathrm{GeV}$. We show a comparison between the default Herwig model and the dynamical strangeness production where we used the LHCtuned parameters (see Tables 3 and 4) and Pythia with the Monash tune

The multiple parton interaction model in Herwig involves two types of subprocesses, hard and soft. Hard processes are allowed to shower and emit quarks and gluons, while soft ones produce only gluons which may not shower. These soft gluons are all colour-connected to each other and the beam remnants, resulting in a single pre-cluster when undergoing

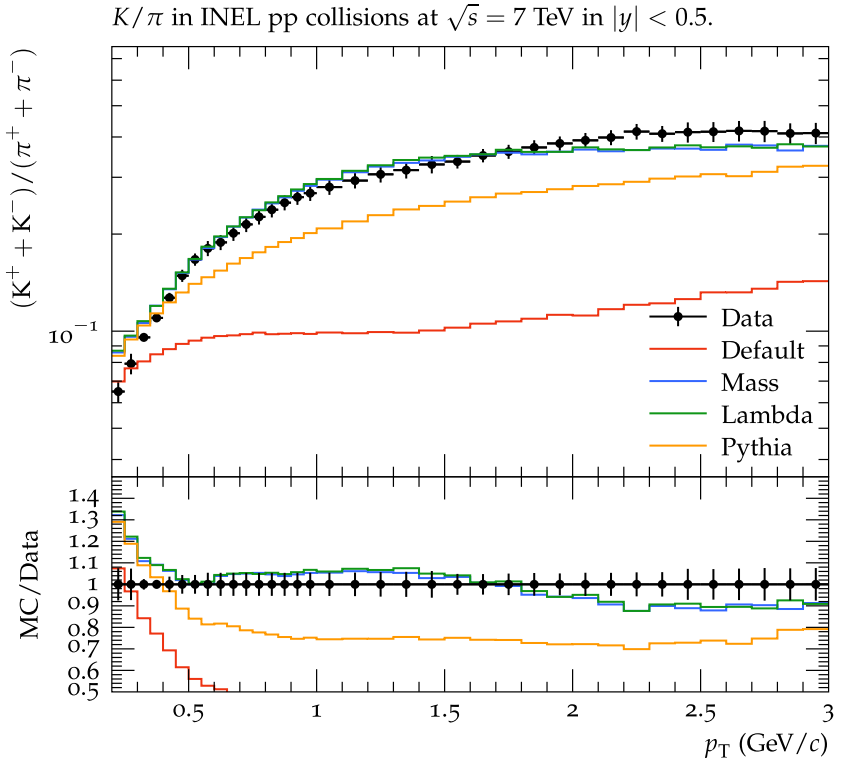

the new approach which introduces dynamical strangeness production with the two different measures (Mass and Lambda) and Pythia with the Monash tune

non-perturbative gluon splitting. This type of pre-cluster typically has a large invariant mass due to the large number of soft gluons and the isotropic nature of their momentum distribution, resulting in a high strangeness production weight for this subsystem. The resulting produced strange particles coming from these soft interactions are distributed uniformly in rapidity.

There are three key differences between the LEP and LHC environments during hadronization. Firstly, LEP has a much lower energy scale than the LHC, naturally limiting the possible distribution of colour-singlet masses at the stage of nonperturbative gluon splittings. As a result, a direct comparison between LEP and LHC in our model is not straightforward.

Secondly, while LEP and LHC simulations may have very similar cluster mass distributions, the number of clusters is far higher for the latter. Similarly, at the pre-cluster level, LEP prefers colour-singlets that span the entire final state, as shown in Fig. 4, i.e. no perturbative gluon splittings during the parton shower. This results in the majority of events either having enhanced strangeness production or none at all, at the gluon splitting level, meaning that a flat weight at this level in hadronization can be justified for LEP runs.

Finally, and related to the previous two, LEP is a much cleaner environment. For lepton collisions, there are no multiple parton interactions, nor much effect from colour reconnection. However, in proton collisions, these are both vital phases of the simulation that drastically change the mass topology of the event. 
Table 5 Expected value of strangeness production weight of our new model in LEP events at $91.2 \mathrm{GeV}$, comparing the total invariant mass results with the $\lambda$ measure results

\begin{tabular}{lll}
\hline$E\left(w_{s}\right)$ at LEP & Mass & $\lambda$ \\
\hline Gluon Splitting & 0.096 & 0.164 \\
Cluster Fission & 0.297 & 0.166 \\
Cluster Decay & 0.009 & 0.016 \\
\hline
\end{tabular}

Table 6 Expected value of strangeness production weight of our new model in LHC Minimum Bias events at $7 \mathrm{TeV}$, comparing the total invariant mass results with the $\lambda$ measure results

\begin{tabular}{lll}
\hline$E\left(w_{s}\right)$ at LHC & Mass & $\lambda$ \\
\hline Gluon Splitting & 0.555 & 0.571 \\
Cluster Fission & 0.018 & 0.020 \\
Cluster Decay & 0.153 & 0.041 \\
\hline
\end{tabular}

Taking the characteristic mass scales from Tables 3 and 4, we have translated these into an effective expected value for the weights for the two mass measures. For LEP events, as shown in Table 5, the total invariant mass approach prefers cluster fissioning, while for the $\lambda$ measure, non-perturbative gluon splitting and cluster fissioning are approximately the same. It should be noted that aside from the gluon splitting weights, there is no direct translation between the kinematic picture and the old model of strangeness production, but these expected values give an idea of the average weights. For gluon splitting at LEP, the weight simply varies between 0 and the maximal value, since pre-clusters are predominately situated around two peaks, as shown in Fig. 4, and the value shown in Table 5 is simply half the maximal value of 0.192 in the invariant mass case, and 0.328 for the $\lambda$ measure.

For LHC Minimum Bias events, the expected value for the weights are shown in Table 6 . There is very little difference between using the two mass measures at the gluon splitting and cluster fission stages, while cluster decay is significantly suppressed when using the $\lambda$ measure. The enormous suppression of strangeness production during the later stages of hadronization compared to the gluon splitting is almost certainly a hint that colour reconnection plays a nontrivial role in producing strange hadrons. Our new kinematic model uses a mass-based scaling, but colour reconnection aims to lower the cluster masses to some local minimum, meaning that it is in direct conflict with our considerations. For LEP simulations, colour reconnection has a small effect, while in LHC simulations, colour reconnection is a vital phenomenon. Future work will study the correlations between the role colour reconnection plays and our model, in particular, varying the amount of colour reconnection that takes place in an event, and allowing baryonic clusters to form.
Our studies showed that there is virtually no quantitative difference between using the tuned invariant mass parameters and the tuned $\lambda$ measure parameters. However, the results in Tables 5 and 6 suggest that the $\lambda$ measure bridges the divide between the two types of collision better.

We have also compared the results of our new model with Pythia and the Monash tune in Figs. 10 and 11. While the Monash tune aims to describe a number of observables other than the strangeness production rate in Pythia, it is tuned to both LEP and LHC data [14], making it an apt benchmark for this discussion.

We can see that our model performs marginally better than Pythia, and significantly better than default Herwig, when trying to describe the $K^{ \pm}$and drastically better on both counts for the $K / \pi$ ratio yields, as shown in Fig. 10 . However, in the low- $p_{\perp}$ region, both Pythia and our model overestimate the data. When using LHC Minimum Bias tuned parameters for LEP simulations, our model outperforms the default Herwig model, but Pythia describes the data better, as shown in Fig. 11.

We expect that changing non-perturbative strangeness production scaling should not change the overall event-shape observables, such as the Sphericity, and total jet broadening. We have included several of these observables from ALEPH data $[20,21]$ in Fig. 12, to confirm that there are only minor statistical differences between default Herwig 7 and our new scaling when one is concerned with non-species specific observables.

While we have not fully solved the discrepancy between the weights for LEP and LHC strangeness production, we have achieved two results: firstly, we have narrowed the gap between the weights of the two types of collision, and in particular, our model can be used with LHC Minimum Bias tuned parameters to better describe LEP data. Secondly, we have made the first steps to a more sophisticated treatment of hadronization and pair production at the low-energy scale in Herwig.

\section{Conclusion and outlook}

We have introduced a three-part model that scales the probability for strangeness production during the hadronization phase of event generation in Herwig. The scaling is directly controlled by the mass of the corresponding event coloursinglet subsystem at each step. With this mechanism, we allow for greater fluctuations in the production of strange pairs on an event-by-event basis.

We have studied the mechanism for non-perturbative strangeness production in detail and found that the current flat probability model is irreconcilable with both LEP and LHC data. A hadronization model should be able to have 

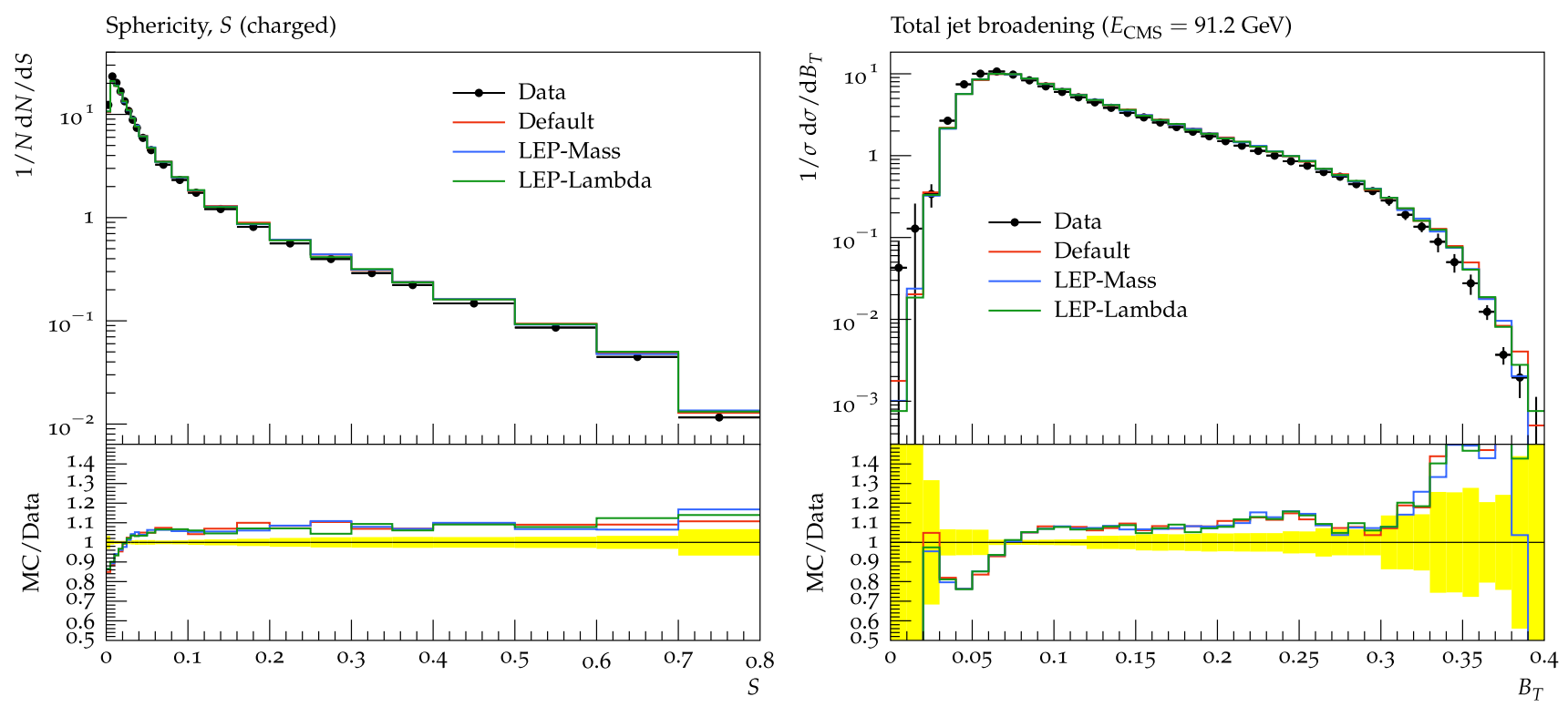

Fig. 12 Event-shape observables from ALEPH [20,21], comparing the results of default Herwig to our new LEP tuned non-perturbative strangeness production scaling, for both mass and $\lambda$ measures. The new scaling does not impact on event-shape observables

minimal effects on LEP simulations, but produce significant effects for LHC simulations.

After allowing a mass-based scaling, and tuning the parameters to LEP and LHC data, we find that we are able to narrow the gap between the two collider types, and able to describe some observables better than the Lund string model in Pythia with the Monash tune. We also provide expected values for non-perturbative strangeness production, which capture the average values for event-by-event fluctuations.

It should be noted that we have not considered heavier hyperons, the production of which has been shown to be increased by creating baryonic clusters at the colour reconnection stage [8]. Baryonic clusters, which are heavier by nature, would modify our model's strangeness production rates. Understanding the interplay between our new model and colour reconnection will be left for future work.

There is still much left to understand in soft physics, but understanding the correlations created between the various models in hadronization are imperative to having more precise and useful Monte Carlo event generators.

Acknowledgements We would like to thank Stefan Gieseke and Peter Skands for their comments on the manuscript. We would also like to thank Stefan Prestel for his helpful comments on the work and Peter Christiansen for his invaluable help understanding experimental analyses. This work has received funding from the European Union's Horizon 2020 research and innovation programme as part of the Marie Sklodowska-Curie Innovative Training Network MCnetITN3 (grant agreement no. 722104). This work has been supported by the BMBF under Grant number 05H18VKCC1.

Data Availability Statement This manuscript has no associated data or the data will not be deposited. [Authors' comment: The data sets were generated with Herwig 7.1.4, an update to the currently available
Herwig 7.1.3. The work presented in this paper will be published in the future release of 7.1.4. However, the code and the data maybe provided on reasonable request from either of the authors.]

Open Access This article is distributed under the terms of the Creative Commons Attribution 4.0 International License (http://creativecomm ons.org/licenses/by/4.0/), which permits unrestricted use, distribution, and reproduction in any medium, provided you give appropriate credit to the original author(s) and the source, provide a link to the Creative Commons license, and indicate if changes were made.

Funded by SCOAP $^{3}$.

\section{References}

1. ALICE Collaboration, J. Adam et al. Nature Phys.13 535-539 (2017). arXiv: 1606.07424

2. C.M.S. Collaboration, V. Khachatryan, JHEP 05, 064 (2011). arXiv: 1102.4282

3. C. Bierlich, G. Gustafson, L. Lönnblad, A. Tarasov, Tarasov JHEP 03, 148 (2015). arXiv: 1412.6259

4. C. Bierlich, G. Gustafson, L. Lönnblad. arXiv:1612.05132

5. T. Pierog, I. Karpenko, J.M. Katzy, E. Yatsenko, K. Werner. Phys. Rev. C 92(3), 034906 (2015). arXiv:1306.0121

6. B. Blok, C.D. Jäkel, M. Strikman, U.A. Wiedemann, JHEP 12, 074 (2017). arXiv:1708.08241

7. J.R. Christiansen, P.Z. Skands, JHEP 08, 003 (2015). arXiv:1505.01681

8. S. Gieseke, P. Kirchgaeßer, S. Plätzer, Eur. Phys. J. C 78(2), 99 (2018). arXiv: 1710.10906

9. J. Bellm, Eur. Phys. J. C 76(4), 196 (2016)

10. T. Sjöstrand, S. Ask, J.R. Christiansen, R. Corke, N. Desai, P. Ilten, S. Mrenna, S. Prestel, C.O. Rasmussen, P.Z. Skands, Comput. Phys. Commun. 191, 159-177 (2015). arXiv: 1410.3012

11. T. Gleisberg, S. Hoeche, F. Krauss, M. Schonherr, S. Schumann, F. Siegert, J. Winter, JHEP 02, 007 (2009). arXiv:0811.4622

12. B. Andersson, G. Gustafson, G. Ingelman, Phys. Rep. 97(2), 31145 (1983) 
13. N. Fischer, T. Sjöstrand, JHEP 01, 140 (2017). arXiv:1610.09818

14. P. Skands, S. Carrazza, J. Rojo, Eur. Phys. J. C 74(8), 3024 (2014). arXiv: 1404.5630

15. B. Webber, Nuclear Phys. B 238(3), 492-528 (1984)

16. D. Amati, G. Veneziano, Phys. Lett. B 83(1), 87-92 (1979)

17. A. Buckley, H. Hoeth, H. Lacker, H. Schulz, J.E. von Seggern, Eur. Phys. J. C 65, 331-357 (2010). arXiv:0907.2973

18. A. Buckley, J. Butterworth, L. Lonnblad, D. Grellscheid, H. Hoeth, J. Monk, H. Schulz, F. Siegert, Comput. Phys. Commun. 184, 2803-2819 (2013). arXiv:1003.0694

19. S. Gieseke, C. Rohr, A. Siodmok, Eur. Phys. J. C 72, 2225 (2012). arXiv: 1206.0041
20. ALEPH Collaboration, R. Barate et al. Phys. Rept. 294 1-165 (1998)

21. The ALEPH Collaboration. Eur. Phys. J. C Particles Fields. 35 457-486 (2004)

22. DELPHI Collaboration, P. Abreu et al. Z. Phys. C 73 11-60 (1996)

23. S.L.D. Collaboration, K. Abe et al., Phys. Rev. D 69, 072003 (2004). arXiv:hep-ex/310017

24. Particle Data Group Collaboration, C. Amsler et al. Phys. Lett. B 667 1-1340 (2008)

25. ALICE Collaboration, J. Adam et al. Eur. Phys. J. C 75(5), 226 (2015). arXiv:1504.00024 